\title{
LOW EMITTANCE LATTICE FOR LEP
}

\author{
Y. Alexahin, JINR, Dubna, Russia, \\ D. Brandt, K. Cornelis, A. Hofmann, J.P. Koutchouk, M. Meddahi, G. Roy and A. Verdier, \\ CERN, CH-1211 Geneva 23, Switzerland
}

\begin{abstract}
In order to obtain the largest luminosity with LEP2, it is attractive to make the beam emittance as small as possible because the beam-beam effect is not a limitation at the energy of $E \approx 90 \mathrm{GeV}$ for the obtained bunch currents. This can be achieved with a high tune lattice. Two possible candidates are lattices with a horizontal phase advance of $108^{\circ}$ or $135^{\circ}$ per cell. Both have a vertical phase advance of $60^{\circ}$. These lattices were developed during 1994 and the results are presented. Tests to reach high intensity for the $108^{0}$ lattice were performed and the bunch current achieved is compared with expectations. For this lattice the detuning v.s intensity and several optics parameters were measured as well.
\end{abstract}

\section{INTRODUCTION}

The LEP machine was designed for cell phase advances of $60^{\circ}$ and $90^{\circ}$. The resulting transverse beam emittance allows reaching the beam-beam limit at the energy of $85 \mathrm{GeV}$ or higher only with bunch currents considerably larger than the ones possible now. It is therefore beneficial to reduce the emittance of the beam to optimize the luminosity. The smallest emittance is obtained for a cell phase advance of about $135^{\circ}$. The significant luminosity gain to be expected from a smaller emittance is enhanced by the smaller beam size at the collimators close to the low- $\beta$ quadrupoles, allowing to decrease $\beta^{*}$ and thus further increase the luminosity [1]. Unlike synchrotron light sources, strong chromatic aberrations are generated by the low- $\beta$ insertions which have to be corrected by the cell sextupoles. To avoid a too strong perturbation to the beam motion, they must be arranged in pairs at an odd multiple of $180^{\circ}$, constraining the cell phase advance to $90^{\circ}, 108^{\circ}$ or $135^{\circ}$, [2]. The reduced dispersion function demands an increased strength of the sextupoles which enhances the non-linear perturbation to the beam motion. Fortunately the largest chromatic aberration is in the vertical plane, where the cell phase advance can be kept low, thereby reducing somewhat the sextupole excitation. The stronger focusing shortens the bunch length and decreases the threshold for the transverse mode coupling instability. Conversely, the RF bucket is larger, making it possible to slightly increase the beam energy in a critical range. The ultimate gain in luminosity reaching 3.5 , a theoretical and experimental study was initiated to investigate the potential of a minimum emittance lattice.

\section{HIGH TUNE LATTICES}

\section{A. $135 / 60$ lattice.}

In this lattice the arc FODO cells have a horizontal phase advance of $135^{\circ}$ and a vertical phase advance of $60^{\circ}$. The horizontal tune is 125.28 and the vertical tune is 75.18 . Given the machine super-periodicity, such tunes guarantee that no problem is expected from the non-linear chromaticity and two sextupole families are sufficient [3].

Since early runs in 1993 could not obtain a circulating beam the problems associated with this lattice were studied extensively [4] with the outcome that it is not possible to correct both the third order resonances and the derivative of the horizontal tune with respect to the horizontal emittance. The latter has a value of $1.6 \times 10^{5} \mathrm{~m}^{-1}$ for the lattice under consideration. For an rms closed orbit amplitude of $4 \mathrm{~mm}$ at the BPM's where $\beta_{x}$ is about $10 \mathrm{~m}$, the associated emittance is about $1.6 \mu \mathrm{rad} . \mathrm{m}$, which makes an associated horizontal tune-shift of -0.26 . As the fractional part of the horizontal tune of our machine is 0.28 , we see that a badly corrected closed orbit can easily lead to a linear instability. In addition, the orbit excursions in the sextupoles lead to a widening of the second order stop-band which can reach a width of 0.15 for an rms closed orbit deviation of $0.5 \mathrm{~mm}$.

In practice it can be observed that the horizontal tune, estimated from a Fourier analysis of the measurements over several turns, wanders considerably depending on the trajectory corrections applied. It is important to note that applying a tune-shift does not help because of these large tune changes.

In 1994 great care was taken to correct the first turn trajectory so that its r.m.s. value was between 3 and $2 \mathrm{~mm}$. Then the orbit closure algorithm [5] was applied and a circulating beam was obtained after having applied it iteratively and having made a systematic horizontal tune-shift of about +0.2 in order to compensate for the anharmonic effects. Another experiment devoted to accumulate more current had various troubles and only $59 \mu \mathrm{A}$ were stored with the damping wigglers on. This is not limited by collective effects.

\section{B. $108 / 60$ lattice}

In 1994, in parallel with the analysis of the problems of the $135^{\circ} / 60^{\circ}$ lattice, a $108^{\circ} / 60^{\circ}$ lattice was developed from the $90^{\circ} / 60^{\circ}$ lattice of 1994 , which was the one used for operating LEP at this time. This $108^{\circ} / 60^{\circ}$ lattice has now two horizontal and three vertical sextupole families. It was developed for machine developments purposes and is not yet optimized for pretzel or bunch train operation. The derivative of the horizontal tune with respect to the horizontal emittance was $2.2610^{4} \mathrm{~m}^{-1}$ the one in the vertical plane was $7.3710^{4} \mathrm{~m}^{-1}$, and the cross term (derivative of the vertical tune with respect to the horizontal emittance) was $-7.9710^{4} \mathrm{~m}^{-1}$. For a given orbit distortion the maximum detuning is reduced by $50 \%$ compared to the $135^{\circ} / 60^{\circ}$ lattice. Although still large, it is sufficiently reduced to obtain easy injection, accumulation in the first experiment.

Five experiments were performed in order to test the performances of the $108^{\circ}$ lattice. They were very successful and proved the easy operation of the machine with this lattice, from the in- 
jection, accumulation to the ramp and squeeze. Various measurements were done which are developed in the following chapter.

\section{Collective effects}

One of the problems encountered by the high tune lattices is the small momentum compaction factor $\alpha_{e}$. The smaller emittance is obtained by strong focusing resulting in small values of the horizontal dispersion function $D_{x}$ and consequently in a reduction of the transverse quantum excitation by the emitted synchrotron radiation. This reduces also the momentum compaction $\alpha_{c}$ and the synchrotron tune $Q_{s}$. The bunch current in LEP is limited by the transverse mode coupling instability (TMCI). The tunes of the different head-tail modes are separated at small current by $Q_{s}$. At larger current the transverse impedance causes tune shifts which can bring two modes together resulting in an instability. Obviously, a large basic separation $Q_{s}$ of the modes gives a higher TMCI threshold. The effective impedance involved tends to increase with reduced bunch length $\sigma_{s}$. Since increasing $Q_{s}$ will at the same time decrease $\sigma_{s}$, the maximum bunch current improves only slowly with $Q_{s}$ as shown in Fig. 1. Some gain in current can be obtained with wigglers located in dispersion free sections. They increase the energy spread of the beam and therefore also the bunch length. Two groups of such wigglers are available in LEP called damping (DW) and polarization (PW) wigglers according to their original purpose. The beneficial effect of these devices is also indicated in Fig. 1.

\section{Phase advance in the vertical plane}

The choice of $60^{\circ}$ vertical phase advance per cell was motivated by the need to guarantee a good non-linear chromaticity correction and also a good vertical orbit correction for polarization. This is not without consequence for the collective effects. Moving from $90^{\circ}$ to $60^{\circ}$ in the vertical plane increases the average vertical $\beta$-function in the arcs by up to $30-40 \%$ when averaging over all the bellow positions in the arcs (e.g. when moving from a $90^{\circ} / 90^{\circ}$ to a $135^{\circ} / 60^{\circ}$ lattice). Since for the present bunch length the bellows represent about half of the total impedance in the vertical plane, the resulting reduction of the maximum intensity at injection (at fixed $Q_{s}$ ) would thus amount to $15-20 \%$. This is not negligible and could even annihilate the gain expected from the reduced emittances. In the case where a vertical phase advance of $90^{\circ}$ is not ruled out by polarization considerations, a $108^{\circ} / 90^{\circ}$ optics $\left(<\beta_{y}>=71.0 \mathrm{~m}\right.$ in the arcs) would become a very interesting candidate for LEP2.

\section{COMPARISON OF THE DIFFERENT OPTICS}

A comparison between the different optics presently considered for the operation of LEP2 is presented in Table I. It has been assumed that average $\beta$ value in the straight sections can be kept the same for all three optics and we concentrate on the variations in the arcs. Similarly, only the horizontal emittance is quoted, since the ultimate value of the vertical one will strongly depend on our ability to correct both the dispersion and the coupling.

\begin{tabular}{|cc|c|c|c|}
\hline \multicolumn{2}{|c|}{ Parameter } & $90^{0} / 60^{0}$ & $108^{0} / 60^{0}$ & $135^{0} / 60^{0}$ \\
\hline \multicolumn{2}{|c|}{ integer $\mathrm{Q}_{x}$} & 90 & 102 & 125 \\
\multicolumn{2}{|c|}{ integer $\mathrm{Q}_{y}$} & 76 & 76 & 75 \\
\multicolumn{2}{|c|}{ mom. comp. $\alpha$} & 0.000186 & 0.000138 & 0.000102 \\
$\beta_{x}$ in QF & {$[\mathrm{m}]$} & 122.0 & 130.2 & 178.5 \\
$\beta_{x}$ in QD & {$[\mathrm{m}]$} & 25.5 & 18.2 & 10.2 \\
$\beta_{y}$ in QF & {$[\mathrm{m}]$} & 41.0 & 38.7 & 36.5 \\
$\beta_{y}$ in QD & {$[\mathrm{m}]$} & 152.7 & 162.3 & 175.3 \\
$\left\langle\beta_{x}\right\rangle$ in arcs & {$[\mathrm{m}]$} & 64.1 & 63.2 & 75.4 \\
$\left\langle\beta_{y}\right\rangle$ in arcs & {$[\mathrm{m}]$} & 85.7 & 87.6 & 92.5 \\
$\mathrm{D}_{x}$ in $\mathrm{QF}$ & {$[\mathrm{m}]$} & 1.13 & 0.88 & 0.68 \\
$\mathrm{D}_{x}$ in $\mathrm{QD}$ & {$[\mathrm{m}]$} & 0.60 & 0.42 & 0.28 \\
$\epsilon_{x}(90 \mathrm{GeV})$ & {$[\mathrm{nm}]$} & 45.6 & 29.6 & 22.8 \\
$\sigma_{s}(90 \mathrm{GeV})$ & {$[\mathrm{mm}]$} & $\approx 11.0$ & $\approx 8.2$ & $\approx 6.0$ \\
\hline \multicolumn{4}{|c|}{}
\end{tabular}

Table I

Comparison between different LEP2 optics

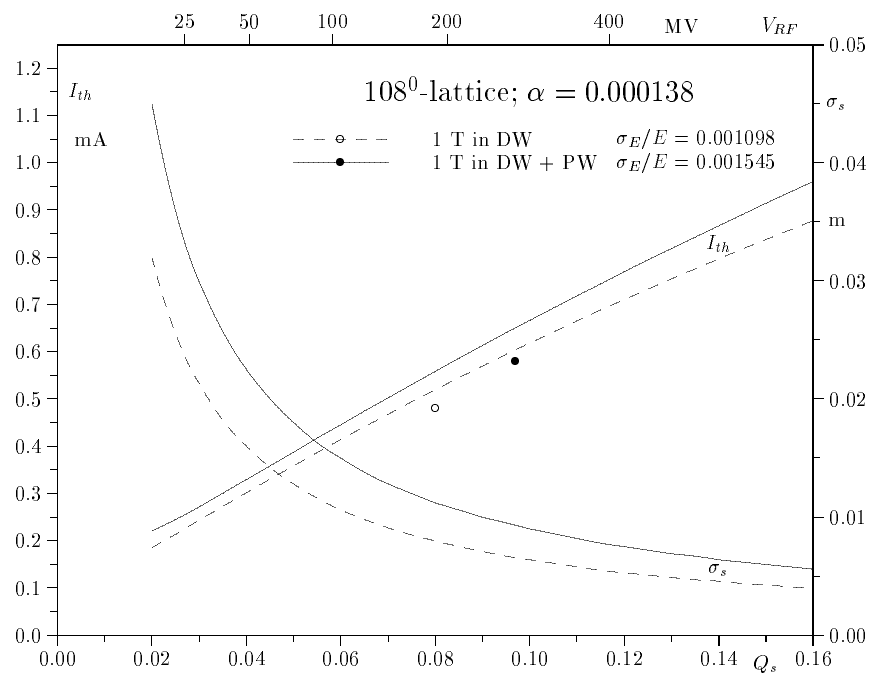

Figure. 1. Calculated and measured TMCI thresholds vs. $\mathrm{Q}_{s}$ for two settings of damping and polarization wigglers DW and PW

\section{RESULTS FROM EXPERIMENTS FOR THE $108^{\circ}$ LATTICE}

\section{A. Current limitations and high $Q_{s}$}

With the damping wigglers alone and a synchrotron tune of 0.08 , the maximum intensity reached was $480 \mu \mathrm{A}$ per bunch. This limit was due to transverse mode coupling instabilities and is not much smaller than the value obtained with the $90^{\circ} / 60^{\circ}$ lattice, under the same conditions. Using the polarization wigglers in addition and with a $Q_{s}=0.093$, the maximum intensity reached 560 $\mu \mathrm{A}$ per bunch. Increasing $Q_{s}$ to 0.097 gave a maximum intensity of $580 \mu \mathrm{A}$ per bunch, which agrees well with the current of $620 \mu \mathrm{A}$ predicted by simulation for the TMCI as shown in Fig. 1. This value is also very close to the single beam limit of $630 \mu \mathrm{A}$ obtained presently with the $90^{\circ} / 60^{\circ}$ lattice for the same $Q_{s}$ [6]).

The changes of the betatron tunes with bunch current are good measures for the effective transverse impedances and were mea- 


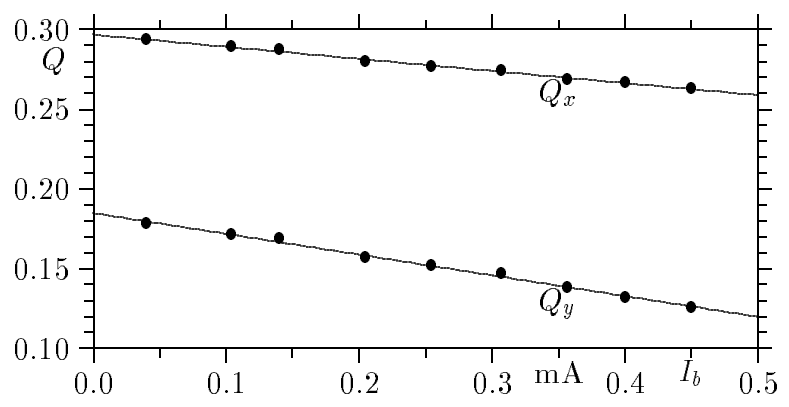

Figure. 2. Measured tune change with bunch current

\begin{tabular}{|c|c|c|c|c|}
\hline$\Delta f_{r f}(\mathrm{~Hz})$ & $Q_{x}$ & $Q_{y}$ & $\epsilon_{x}(\mathrm{~nm})$ & $\epsilon_{y}(\mathrm{~nm})$ \\
\hline-50 & .259 & .181 & 12.15 & 1.31 \\
0 & .258 & .179 & 9.43 & 1.24 \\
+50 & .257 & .177 & 7.79 & 1.28 \\
\hline
\end{tabular}

Table II

Measured tunes and emittances for different RF-frequencies

sured to be $d Q_{x} / d I_{b}=-77 \mathrm{~A}^{-1}$ and $d Q_{y} / d I_{b}=-130 \mathrm{~A}^{-1}$, Fig. 2 . which are comparable with those of the $90^{\circ} / 60^{\circ}$ lattice.

\section{B. Optics and emittance measurements}

During the last 1994 experiment, the beam was ramped to 46 $\mathrm{GeV}$. During the ramp, it was observed that each horizontal orbit correction led to a change of both tunes caused by the large sextupole strength as expected. This was as well the case after the ramp and during the squeeze. After few iterations of orbit corrections, the chromaticities were measured to be $Q_{x}^{\prime}=1.1$ and $Q_{y}^{\prime}=1.6$. The vertical rms dispersion was found to be $6 \mathrm{~cm}$.

The emittance, being a significant parameter of this lattice, was measured with the synchrotron light monitor BEUV [7] for different RF-frequencies. The obtained horizontal and vertical emittances are listed in table II. From them, the uncoupled emittance $\epsilon_{0}=\epsilon_{x}+\epsilon_{y}$ (assuming that vertical damping and coupling are unchanged) and the derivative of the longitudinal damping partition are obtained. These results are compared with calculations by the program WIGWAM [8] and listed in Table III. The fact that the measured emittance value is a little larger than the expected one is not surprising. In a high tune lattice modest orbit distortions can create relatively large spurious dispersions which lead to an increase of the emittance. Very good orbit corrections will be necessary to profit from this optics.

\begin{tabular}{|c|c|c|}
\hline & measurement & calculation \\
\hline$\epsilon_{0}[\mathrm{~nm}]$ & 10.67 & 7.85 \\
$\Delta J / \Delta p / p$ & 190 & 240 \\
\hline
\end{tabular}

Table III

Measured and calculated emittance and damping partition derivative at $46 \mathrm{GeV}$
The above measurements were carried out with the injection optics having a vertical $\beta$ function of $\beta_{y}^{*}=0.21 \mathrm{~m}$ at the four interaction points. An attempt was made to reduce this $\beta_{y}^{*}$ in steps to the value of $0.05 \mathrm{~m}$ used in physics runs. Orbit corrections were performed at $0.14 \mathrm{~m}$ and $0.09 \mathrm{~m}$ without problems. The next step (to $0.07 \mathrm{~m}$ ) gave a negative vertical chromaticity and the beam was lost. This could be corrected easily but further trials were postpone due to lack of time. The easiness with which the ramp and partial squeeze could be performed indicates that no problems are expected for the operations of the $108^{\circ} / 60^{\circ}$ lattice.

\section{Conclusions}

High tune lattices giving small emittances are promising means to obtain a high luminosity for LEP operation at $90 \mathrm{GeV}$ where the bunch current is limited by instabilities at injection and not by the beam-beam effect in collisions. Solutions have been worked out and tried experimentally for horizontal phase advances per cell of $135^{\circ}$ and $108^{\circ}$. The first configuration gives about the smallest emittance possible in a regular FODO-lattice. The strong horizontal focussing obtained in these lattices leads to a small synchrotron tune and bunch length for a given RFvoltage. This reduces the threshold for the transverse mode coupling instability. The wiggler magnets available in LEP have to be used to lengthen the bunch and to keep the current reduction within limits. For the $108^{0}$-lattice a bunch current of over 0.6 $\mathrm{mA}$ has been achieved which is close to the expected limit. Furthermore, the emittance measured at $46 \mathrm{GeV}$ is not much above the calculated value. Other parameters have been checked and the $\beta$ function in the interaction points partially reduced. These results clearly show that high luminosity operation of LEP at 90 $\mathrm{GeV}$ is feasible and that the $108^{\circ} / 60^{\circ}$ lattice is a good candidate for LEP2.

\section{References}

[1] J.P. Koutchouk, "A Superlow Emittance for LEP 2", Proc. of the 3rd workshop on LEP performance, edited by J. Poole, p. 511, CERN SL/93-19 (DI), 1993.

[2] J.R.J. Benett et al., "Design Concept for a $100 \mathrm{GeV} \mathrm{e} \mathrm{e}^{+} \mathrm{e}^{-}$ Storage Ring (LEP), CERN 22-14, 1977. 1993 Part. Acc. Conf., Washington D.C., 17-20 May 1993.

[3] A. Verdier, "Higher order tune derivatives due to low- $\beta$ insertions", Proc. 1993 Part. Acc. Conf., Washington D.C., p. 249.

[4] Y. Alexahin, "A study of the low emittance lattice for LEP2”, CERN SL/94-46 (AP), 1994.

[5] A. Verdier and F. Richard, "Algorithms to get a circulating beam", This conference.

[6] D. Brandt and A. Hofmann, "Does a high $Q_{s}$ raise the Maximum intensity to be Accumulated in LEP?", Proc. of the 4th workshop on LEP performance, edited by J. Poole, $p$. 149, CERN SL/94-06 (DI), 1994.

[7] G. Baribaud, C. Bovet, R. Jung, M. Placidi, E. Rossa and Y. Solberg, "Three Dimensional Bunch Observation in LEP", Proc. of th 15th Intern. Conf. on High Energy Accelerators, Hamburg 1992, p. 212.

[8] J.M. Jowett, "LEP2 Beam Parameters during Energy Ramping", CERN LEP2 Note 95-27, 1995. 